\title{
Engineers as Life-Long Learners: Pedagogical ToOls For ENGINEERING GRADUATE ATTRIBUTE DEVELOPMENT
}

\author{
Shermeen Nizami; Mohamed Abdelazez; Adrian D.C. Chan \\ Department of Systems and Computer Engineering, Carleton University, Ottawa, Canada \\ shermeen@sce.carleton.ca,mohamedabdelazez@cmail.carleton.ca,adcchan@sce.carleton.ca
}

\begin{abstract}
We integrate three pedagogical constructs to design a novel toolbox to support engineering graduate attribute development and measurement of life-long learning. These constructs are (i) Bloom's Taxonomy of Educational Objectives in the Cognitive Domain, (ii) Curriculum mapping to skill levels of Introduction, Reinforcement, Mastery and Assessment (IRMA), and (iii) High Impact Practices (HIPs). We use the toolbox in a single, but in-depth, experiential education research study in our department's Biomedical and Electrical Engineering program. We formally introduce these pedagogies to a cohort of eighteen final year students. The students then use the toolbox throughout the term to identify and address their own educational needs such that they develop into independent, competent life-long learners. We test the effectiveness of the toolbox using mixed methods research. Bloom's Taxonomy levels scored by the instructor and the students had a correlation coefficient of 0.36. IRMA mapping was tested using binomial hypothesis testing. The test accepted the alternative hypothesis H1: Student mapping of course learning outcomes to a skill level was informed by the instructor's pedagogy and followed the same distribution as the instructor's mapping, i.e., the mapping was not random and $p \neq 0.5$. Results showed $H 1:\{I(47 \%), R(84 \%)$, $M(63 \%)\}$. In addition, experiential impressions of lifelong learning on the students were gathered through qualitatively written feedback.
\end{abstract}

Keywords: engineering graduate attribute; Canadian Engineering Accreditation Board; life-long learning; experiential education; quality assurance, Bloom's Taxonomy; design thinking; curriculum mapping; high impact practices.

\section{INTRODUCTION}

Canadian Engineering Accreditation Board (CEAB) evaluates Canadian engineering programs for the purpose of accreditation based on Graduate Attribute (GA) measures. The institution must demonstrate that the program has inculcated in its graduates twelve attributes specified by the CEAB. These attributes are interpreted in the context of candidates at the time of graduation with the recognition that graduates will continue to build on the foundations that their engineering education has provided [1]. Earlier work at Carleton University [2] proposes a high-level "Graduate attributes" process that guides departments to align their program outcomes to meet GA requirements. This process is informed by the Engineering graduate attribute development (EGAD) project's five step guide to curriculum development [3]. The process in [2] elaborates on how to (i) identify learning objectives, (ii) create measurable criteria for different graduate attributes, (iii) map the criteria to program outcomes, (iv) identify appropriate measures and standards, (v) measure the criteria, (vi) analyze and evaluate the data, and (vii) improve the program based on results. Recent research at Concordia University [4] identifies two important problems that (a) arise in the processes of translation from the CEAB GA to measurable indicators of student performance; and (b) that many traditional engineering professors are ill-equipped to answer. The first problem alludes to the measurement of attributes that create social awareness in our students. For example, "how exactly does one measure how well a student has internalized the "ethics and equity' attribute or the 'life-long learning' attribute?" [4]. The second observed problem is that "many instructors would use existing course syllabi as sources of definitions of indicators, assessment criteria, and rubrics for success for the new CEAB graduate attributes and professors believed that their courses were already meeting the outcomes criteria. Thus, existing pedagogy was repackaged and presented as a way of assessing new graduate attributes" [4]. However, the paper negates that the current engineering education culture enables either the instructor or the students to always be mindful of the GAs. Recent research at Queen's University [5] suggests that a positive culture change can occur given efficient and effective communication between all stakeholders. The institutional approach to planning and assessing GA development should be an outcomes-based process, one that is continuously improved by evidence-informed changes [5].

In this paper, we present experiential education research that demonstrates early adoption of the process developed at Carleton in [2]. Using mixed methods

S. Nizami, M. Abdelazez, \& A.D.C. Chan, Engineers as life-long learners: Pedagogical tools for engineering graduate attribute development, CEEA15, McMaster University, May 31 - June 3, 2015 


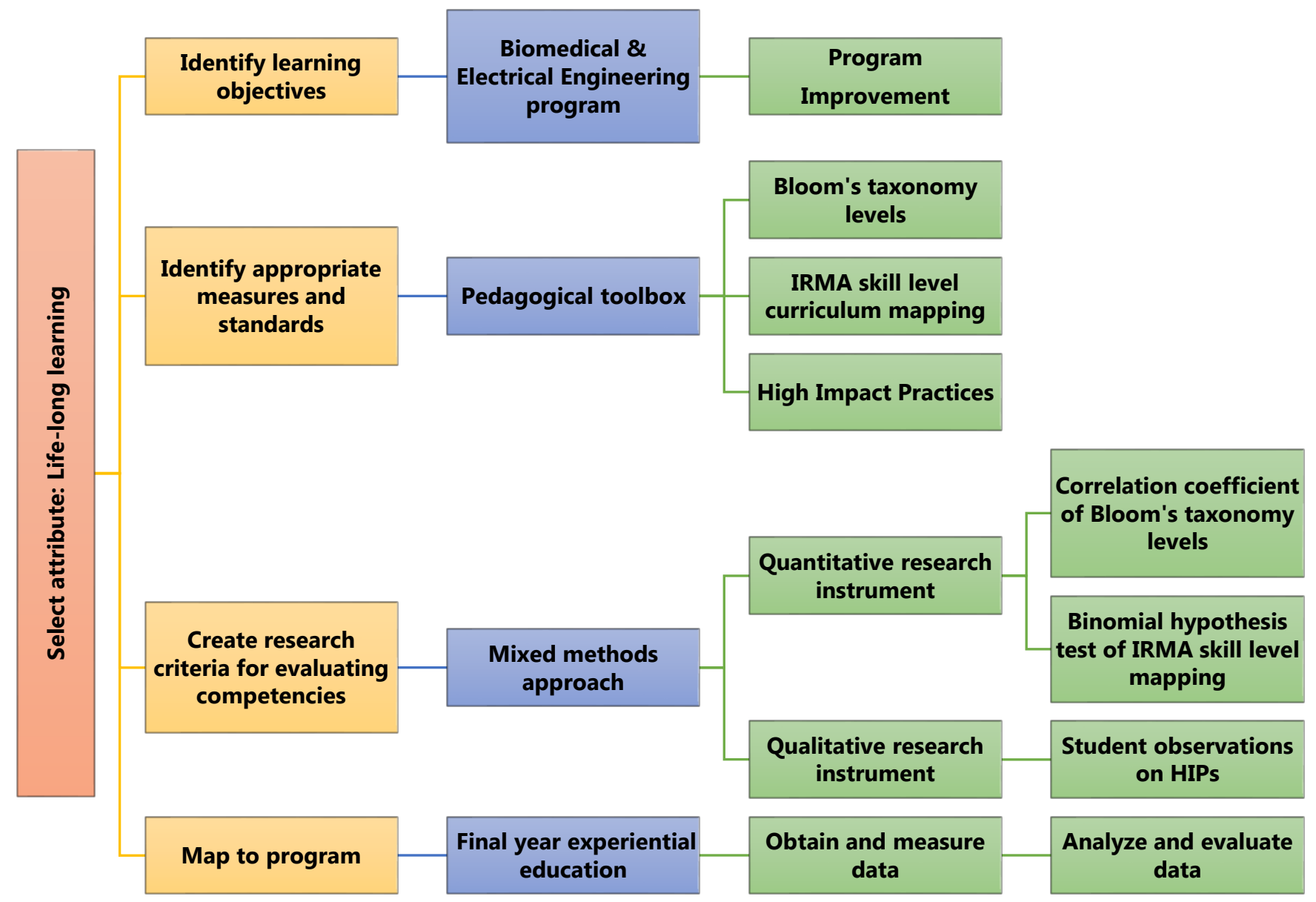

Fig.1. Mixed methods implementation of graduate attribute development and measurement process

research, we enhance the quantitative close-ended process by adding a concurrent qualitative open-ended research dimension to it, as shown in Fig.1. We also present solutions to the two problem identified in [4]. Out of the twelve GAs, we select the attribute of life-long learning to be the required program outcome in our research. CEAB defines the life-long learning attribute in graduates as " $A n$ ability to identify and to address their own educational needs in a changing world in ways sufficient to maintain their competence and to allow them to contribute to the advancement of knowledge" [1]. It's postulated that lifelong learning which is listed last amongst the twelve CEAB's GAs culminates all the others and projects graduates forward into their professional lives [6].

Research from University of Calgary [7] previously used the Likert-type Self-Directed Learning Readiness Scale $^{l}$ as the research instrument for a close-ended assessment of life-long learning in a first year course. Their results are a snapshot of a single use of the self-report instrument and are representative of the attribute in a young adult population with limited exposure to post-secondary education [7]. Use of the questionnaire was neither mapped to engineering course content nor can these performance criteria emerging in first year be readily extrapolated to program outcome at the fourth year level and thus, would require profound planning for future use. Queen's University has briefly conducted qualitative research on life-long learning in one senior year workshop as part of their ongoing research efforts [8].

Taking a pragmatic approach, we integrate three pedagogical constructs to design a novel toolbox to support GA development and measurement of life-long learning in our engineering graduates. The constructs are (i) Bloom's Taxonomy of Educational Objectives in the Cognitive Domain [9], (ii) Curriculum mapping to skill levels of Introduction, Reinforcement, Mastery and Assessment (IRMA) ${ }^{2}$, and (iii) High Impact Practices (HIPs) [10]. These pedagogies are typically used for course design and/or curriculum development. Hence, their primary use is by instructors. The novelty of our research is not only that we integrate these pedagogies into a single toolbox for instructional purposes, but also that we leverage the

\footnotetext{
${ }^{1}$ http://www.lpasdlrs.com/

${ }^{2} \mathrm{http} / / /$ carleton.ca/viceprovost/assessment-of-learning/curriculummapping-design/how-to-map-your-curriculum/
}

S. Nizami, M. Abdelazez, \& A.D.C. Chan, Engineers as life-long learners: Pedagogical tools for engineering graduate attribute development, CEEA15, McMaster University, May 31 - June 3, 2015 
pedagogies to our students for the development and measurement of the life-long learning GA. Although, the development of life-long learning is attributed to the students' entire undergraduate journey. However, this research formally introduces the toolbox to fourth year Biomedical and Electrical Engineering students through experiential education. Firstly, this methodology prudently attunes students' mindfulness to the life-long learning GA. Secondly, it allows for the GA to be interpreted in the context of candidates at the time of graduation. Thirdly, this experiential education is delivered in a fourth year core course in the Biomedical and Electrical Engineering program. Hence, it acts as a potential indicator of the program outcome. Students use the toolbox to build a better understanding of the course design, to participate in improving course design and delivery, and to self-assess their progress through the course. In this way the toolbox enables students to identify and address their own educational needs in ways sufficient to maintain their competence, thus allowing them to contribute to the advancement of knowledge. We postulate that increased understanding and application of learning constructs attributes to skillful life-long learning in individuals. In this research, we evaluate the effectiveness of the toolbox in developing and measuring the life-long learning GA in our Biomedical and Electrical Engineering students.

\section{METHODS}

We take a mixed methods research approach, using both quantitative and qualitative data for a pragmatic understanding of the research problem. Such an approach allows for comprehensive inferences to be made from close-ended measures and open-ended observations in consequence-oriented or problem-centered research [11]. Hence, it is well suited to evaluate indicators that are developed to measure GAs. Fig.1 shows our implementation of mixed methods research for the development and measurement of the life-long learning GA. The following subsections delineate the process shown in Fig.1.

\subsection{Identify Learning Objectives}

We identify the life-long learning GA as the learning objective in the Biomedical and Electrical Engineering program. Results from this research also contribute towards program improvement.

\subsection{Identify Appropriate Measures and Standards}

We build a novel pedagogical toolbox with appropriate measures and standards for the development and measurement of life-long learning. Using constructive research methodology [12], we integrate three pedagogical constructs in the toolbox. One of the constructs is Bloom's Taxonomy of Educational Objectives in the Cognitive Domain. We formally introduce these pedagogies to a cohort of eighteen fourth year students. We use Bloom's Taxonomy to research how engineering students arrange their hierarchical learning from simpler to more complex functions. Students use the taxonomy to classify procedural complexity of engineering tasks. They are expected to continue to develop and use these cognitive skills during life-long learning experiences.

The second construct is curriculum mapping to the four skill levels of Introduction, Reinforcement, Mastery and Assessment. We map course learning outcomes to these IRMA skill levels. Students use these skill levels as indicators of their educational competence. These levels apply not only to course learning outcomes but also to program learning outcomes.

The third pedagogical construct is HIPs. Kuh et al. [10] describe eight essential conditions for HIPs. These are high performance expectations, significant investment of time and effort by students over an extended period of time, interactions with faculty and peers about substantive matters, experiences with diversity, frequent timely and constructive feedback, periodic structured opportunities to reflect and integrate learning, opportunities to discover relevance of learning through real-world applications, and public demonstration of competence. Clearly, these eight conditions attribute to life-long learning. Therefore, we deliver HIPs for experiential education and qualitative data collection and evaluation. Our HIPs include iterative course design and delivery, labs and a term project. HIPs allowed students to work in groups to practice their engineering and communication skills. Peer support was used as a scaffold to enhance life-long learning in the labs and the term project. The aim was to provide students with collegial opportunities to identify and address their educational needs, to maintain their competence amongst peers, and to contribute to the advancement of knowledge collaboratively with their peers. An earlier implementation of the term project was published by the authors in [13].

\subsection{Create Research Criteria for Evaluating Competencies}

Using mixed methods research, we create the following criteria for evaluating the life-long learning GA. The quantitative research instruments are: (i) correlation coefficient of Bloom's Taxonomy levels as scored by the instructor and the students, and (ii) binomial hypothesis testing of IRMA mapping between the instructor and the students.

The instructor ( $\mathrm{SN}$ ) mapped scores from 1 to 5 to each of Bloom's Taxonomy levels with increasing cognitive difficulty. The scoring was $1=$ remember factual 
knowledge, 2 = understand and comprehend the material, 3 = apply knowledge to solve a single-stage problem, 4 = analyze a multi-stage problem by identifying and connecting appropriate strategies, $5=$ evaluate and synthesize ideas and concepts from disparate courses to solve a complex engineering design problem. The instructor assigned a score to each question from the final exam appropriate to the question's cognitive level. These scores were hidden from the students. Students then assigned scores to each question on the final exam using their perception of the cognitive level of difficulty of that question.

Similarly, the instructor mapped skill levels of Introduction, Reinforcement and Mastery to each question from the final exam. This mapping reflected the skill level that the students were expected to have attained in the specific content of that question. Since the skills were mapped to the final exam, hence, it was presumed that the students should be ready at the skill level of being assessed, i.e., Assessment in the IRMA mapping. This mapping remained hidden from the students. Students mapped their own skill levels to each question on the final exam. They ideated that the content relevant to that question was introduced and/or reinforced by the instructor. Following which, they indicated whether or not they were given sufficient instructional and experiential tools in class to attain the skill level of Mastery in the specific content. Binomial hypothesis testing was conducted with the following hypotheses. The null hypothesis was H0: Student mapping of course learning outcomes to a skill level was random with equal probability of being a 0 or 1 , i.e., $\mathrm{p}=0.5$. The alternative hypothesis was H1: Student mapping of course learning outcomes to a skill level was informed by the instructor's pedagogy and followed the same distribution as the instructor's mapping, i.e., the mapping was not random and $\mathrm{p} \neq 0.5$.

Student observations on the use of HIPs are used as the qualitative research instrument. Experiential impressions of students are gathered through unstructured written feedback and student narratives over the entire term. We analyze student observations on the efficacy of HIPs as they attributed to students' life-long learning. As the term progressed, the authors iteratively adapted the course design and delivery in response to student feedback.

\subsection{Map to Program}

This mixed methods research is conducted as part of experiential education in a fourth year Biomedical Engineering course. We obtain quantitative and qualitative student data concurrently as well as sequentially. We then analyze and evaluate these data and make suggestions for improving program outcomes in the context of the selected attribute of life-long learning.

\section{RESULTS}

Two close-ended numerical measures were obtained through quantitative research. Bloom's Taxonomy scoring by the instructor and the students had a correlation coefficient of 0.36 averaged across all 5 taxonomy levels. Hypothesis testing of IRMA skill levels resulted in rejection of $\mathrm{H} 0 . \mathrm{H} 1$ was accepted at the given percentage for each skill: $\{\mathrm{I}(47 \%), \mathrm{R}(84 \%), \mathrm{M}(63 \%)\}$.

Following is a summary of open-ended textual observations made by students on the use of HIPs. These were gathered at various times during the term, at the end of the term, and after a few months following the term. Results presented are contextual to the GA of life-long learning.

Overall, students were satisfied in the directions they found for future course of study and career paths. They could easily relate the course curriculum to real-life applications and to real jobs. Students reflected upon their educational competence using skill level indicators of IRMA. They found the labs to be very useful for hands-on learning, which attributes positively to experiential lifelong learning. Many commented that they were not nervous about the final exam as they were not required to cram information. The HIPs enabled them to retain the content long after the term had ended. Upon graduating from the program, they recommended continued use of the pedagogical toolbox to deliver experiential education to future cohorts. They also wished for more engineering professors to use such innovations in education.

\section{DISCUSSION}

Most students were largely unfamiliar and hence, initially hesitant with the use of the pedagogical toolbox. However, after repeated use, they started to value the lifelong learning skills that it reinforced. Multiplexity of the toolbox helped individuals discover which tools served their learning needs best. They sensed independent learning in these pedagogies. They could utilize the pedagogical tools in multiple ways to absorb information and gain deeper conceptual understanding.

Quantitative results indicate that students may need more time to familiarize themselves with Bloom's Taxonomy. With the instructor guiding them in class, they could identify strategies and integrate prior knowledge to solve engineering problems at Bloom's cognitive levels of application, analysis and synthesis. However, the correlation result arises from their first independent attempt at scoring levels of cognition required for solving a given engineering problem.

Student mapping of course learning outcomes to IRMA skill levels was informed by the instructor's pedagogy and followed the same distribution as the instructor's mapping. Students struggled to recall whether certain content was introduced (I) in this course or it was part of their prior

S. Nizami, M. Abdelazez, \& A.D.C. Chan, Engineers as life-long learners: Pedagogical tools for engineering graduate attribute development, CEEA15, McMaster University, May 31 - June 3, 2015 
knowledge. There was certainly more agreement on which content was reinforced $(\mathrm{R})$ in the course. It is encouraging to see that students were gaining more confidence in identifying content that they had mastered (M) in the program.

Qualitative results reflect how well students internalized the attribute of life-long learning. The collaborative course design iterations definitely served as an underpinning for this GA. As a HIP, the instructor and the students mutually identified what is working in the course and what is not. This resulted in adaptive iterations to the course design and delivery. Again, students were initially cautious in approaching such adaptations. However, soon after they realized that these adaptations served their own learning needs better, and that they were positively impacting their learning. Thereafter, they welcomed this HIP. Efficient, effective and consistent open-ended communication between the authors and the students created a positive culture change in engineering education as evidenced by qualitative data. This enabled the instructor and the students to certainly remain mindful of the life-long GA.

\section{CONCLUSION}

In conclusion, we made evidence-informed changes to continually improve the outcomes-based GA process. The process reinforced delivery of tools to students for competent and independent learning. This resulted in a unique course that challenged students to improve their ability to learn. That, in essence, is the attribute of life-long learning.

We plan to further develop and evaluate our integrated pedagogy in future offerings of this course. For instance, Bloom's Taxonomy scores and IRMA skill levels will be mapped to each type of learning activity from the beginning of the course. Students will be provided with a greater number of opportunities to independently map the scores and skill levels over the course duration. We also recommend that the toolbox be leveraged to students starting in the lower year courses in the program. Repetitive use throughout the undergraduate program will help evaluate the robustness of the toolbox as an indicator of the life-long learning GA.

\section{References}

[1] Canadian Engineering Accreditation Board, "What is the accreditation criteria and procedures report?" Engineers Canada, 2014.

[2] J. Harris, A. Steele and D. Russell, "Progress on defining the CEAB graduate attributes at Carleton University," Proceedings of the Canadian Engineering Education Association, 2011.

[3] B. Frank, S. Fostaty-Young, S. McCahan, P. Ostafichuk, N. Saleh, K. C. Watts and P. Wolf, "Engineering graduate attribute development (EGAD) project," in Canadian Engineering
Education Association Conference (CEEA), St. John's, NL, 2011.

[4] B. Caron, G. Gopakumar, D. Dysart-Gale and M. Harsh, "Navigating a Constitutional Moment: Reflections on Implementing Graduate Attributes in Canadian Engineering Education," Engineering Studies, vol. 6, pp. 44-61, 2014.

[5] J. Kaupp and B. Frank, "Approaching the loop: A review of effective practises in continuous program improvement," in Canadian Engineering Education Association Annual Conference (CEEA), Canmore, BC, 2014.

[6] S. Murphy and S. Nasser, "Information literacy in CEAB's accreditation criteria: The hidden attribute," in Canadian Engineering Education Association Conf. (CEEA), 2011. [7] R. W. Brennan, M. Eggermont, W. Rosehart, A. K. Deacon, N. Larson and T. A. O'Neill, "Assessing life-long learning in a first-year design and communication course ," in Proceedings of the Canadian Engineering Education Association (CEEA), 2014.

[8] N. Saleh and D. Strong, "Students' conceptions of life-long learning: An exploratory study," in Canadian Engineering Education Association (CEEA) Conference, 2010.

[9] L. W. Anderson and D. R. Krathwohl, A Taxonomy for Learning, Teaching and Assessing: A Revision of Bloom's Taxonomy of Educational Objectives. New York: Longman, 2001 .

[10] G. D. Kuh, K. O’Donnell and S. Reed, "Ensuring Quality \& Taking High-Impact Practices to Scale," Association of American Colleges \& Universities, Washington, DC, 2013. [11] J. W. Creswell, Research Design: Qualitative, Quantitative, and Mixed Methods Approaches. Sage publications, 2013.

[12] K. A. Piirainen and R. A. Gonzalez, "Seeking constructive synergy: Design science and the constructive research approach," in Lecture Notes in Computer Science, J. vom Brocke, R. Hekkala, S. Ram and M. Rossi, Eds. Springer Berlin Heidelberg, 2013, pp. 59-72.

[13] A. D. C. Chan and S. Nizami, "Integrative learning through the design of an electrocardiogram acquisition system," in Annual International Conference of the IEEE Engineering in Medicine and Biology Society, EMBC, 2011, pp. 3624-3627.

S. Nizami, M. Abdelazez, \& A.D.C. Chan, Engineers as life-long learners: Pedagogical tools for engineering graduate attribute development, CEEA15, McMaster University, May 31 - June 3, 2015 